\title{
Teleworker's Perception of Technology Use for Collaborative and Social During the COVID-19 Pandemic
}

\author{
Jie Cai, Sarah J Ryu, Donghee Yvette Wohn, Hyejin Hannah Kum-Biocca \\ New Jersey Institute of Technology \\ New Jersey, United States \\ jie.cai@njit.edu; sr984@njit.edu; wohn@njit.edu, hyejin.kum-biocca@njit.edu
}

\begin{abstract}
With the flourish of collaborative and social technologies in the market since the pandemic, there is limited understanding of user's attitudes towards these technologies. We aim to understand teleworkers' perceptions of technology use during the pandemic and interviewed 46 teleworkers. We found that teleworkers generally hold a positive attitude towards social technologies and are creative to use these technologies to meet their social needs; they express overall negative feelings about remote collaboration technologies, though online communication flattens the communication hierarchy in the organization. The pandemic amplifies the extant challenges and highlights the shortcomings of technological design in wellestablished teleworking research and remote collaboration work. We suggest that future design should 1) combine and commercialize solutions that are well-grounded in prior work; 2) consider scenarios that are typically missed and can be easily replaced with collocated interaction from the pre-pandemic context into the forced teleworking context.
\end{abstract}

COVID-19 pandemic, work from home, social and work technology, remote collaboration

\section{INTRODUCTION}

The global coronavirus 2019 (COVID-19) pandemic has led to government-mandated physical distancing and even lockdown in some areas. Many organizations scaled back their business or even ceased operations in the regular workplace, instead, asking employees to work from home (teleworking) to reduce the infection risk (Mattern et al. 2021). Additionally, simulations show that many areas in the world will probably suffer several waves of contagion and recurring lockdown (Kissler et al. 2020). The worldspread pandemic with the government-imposed restriction not only affect healthcare and economies at the collective level, but also change the lifestyles of people at the individual level, and these changes have become the new normal of daily life (Liu et al. 2020).

The pandemic accelerates the trend to migrate to teleworking with appropriate technology use in both social and work contexts and increases our dependency on technology use (Waizenegger et al. 2020). For example, the downloading of video conferencing software jumped $45 \%$ immediately after the early lockdown and reached a $90 \%$ average increase in downloads compared to pre-COVID demand (Sydow 2020); most people reported that their social media consumption and posting have increased during the pandemic (Wold 2021). Though a lot of news and reports show the increase of technology use during the pandemic and the role technology played in transforming businesses or improving the psychological treatment of patients online (Saladino et al. 2020; Nah and Siau 2020), revealing the promising and positive aspect of technology use in this new normal, there is limited knowledge about user's perception about the role of technology played in work collaboration and social interaction during the pandemic. We acknowledge the well-grounded research in telepresence and remote collaboration in Human-Computer Interaction $(\mathrm{HCl})$ and Computer-Supported Corporate Work (CSCW) (e.g., Kuzuoka 1992; Ishii et al. 1993; Brave et al. 1998; Fussell et al. 2004). However, prior work has not been conducted in a pandemic context with forced quarantine and physical distancing. It is still unclear to what extent prior work can apply to this new normal context and what still needs to improve to adapt technology to this context. Thus, it is critical and timely to understand user's perceptions of the current technology use to help researchers 
and designers identify the challenges of technology use in the enforced teleworking environment.

In line with recent work proposing the necessity to review the technology use during the pandemic and considering them continuously used over time or cyclically in the recurring outbreaks (Golinelli et al. 2020), in this paper, we interviewed 46 teleworkers working/socializing during the pandemic to explore how users collaboratively use technology in both social and work contexts and to explore their perceptions and attitudes towards the role that technology played. We show how teleworkers perceive the technology use, praising the positive sides while also highlighting the challenges during the usage. These findings highlight the existing challenges in commercialized collaboration tools in well-established research about teleworking and remote collaboration, support recent work proposing the use of new and positive technology to mitigate COVID-19 impact on individual users, and imply to customize and improve current technical solutions, fulfilling people's needs in different contexts during the pandemic and beyond.

\section{RELATED WORK}

The pandemic accelerates the digital transformation in many domains, such as healthcare and business (e.g., Golinelli et al. 2020; Nah and Siau 2020). In this section, we focus on teleworkers' experience and perception towards technology use in remote collaboration and socialization.

\subsection{Teleworking and COVID-19}

Prior work on teleworking has discussed a wide range of issues such as work-life boundary management (Fonner and Stache 2012), advantages (e.g., perceived autonomy, job satisfaction, performance, and role stress) and disadvantages (e.g., feelings of alienation, isolation, worry) of teleworking (Collins 2005; Gajendran and Harrison 2007), and technological impact on teleworking (Cox et al. 2014).

The role of technology in teleworking can be both positive and negative. For example, mobile technology allows people to work remotely with flexible hours that fit around caring responsibilities; it also results in an "always-online" culture that can disrupt work-life balance and induce stress regarding constant connectivity and responsiveness (Cox et al. 2014). Stawarz et al. (2013) found that though tablets can facilitate both home and work tasks, they can potentially blur the work-life boundary by encouraging people to do work tasks during home time and vice versa. Prior work also suggests that controlling working hours is the most critical ability to achieve a positive work-life balance (Maruyama et al. 2009), but teleworkers grapple with the tension between the desire for flexibility and the need to maintain the work-life boundary (Fonner and Stache 2012).

Olson and Olson (2000) explain that effective teleworking in the sociotechnical condition is determined by four key components: common ground, coupling/dependency of work, collaboration readiness, and collaboration technology readiness. They suggest that groups, with high common ground and loosely coupled work, with readiness both for collaboration and collaboration technology, have the potential to succeed with teleworking, and deviations from each component restrict teammates' performance and require changes during the collaboration.

Different from regular teleworking, the pandemic quarantines people, forces people to work from home, restricts mobility, and enforces physical distancing; thus, the enforced teleworking causes challenges to communication and personal well-being (Waizenegger et al. 2020). Forced teleworking might change the interaction dynamics of the four components by Olson and Olson (2000). For example, teleworkers need to adapt quickly to unfamiliar digital technologies to maintain regular business and communication. The enforced teleworking with high intensity can deteriorate the relationships with coworkers as well (Gajendran and Harrison 2007).

\subsection{Remote Collaboration and Technology Use during COVID-19}

Remote collaboration research is well-grounded in both $\mathrm{HCl}$ and $\mathrm{CSCW}$. Though distance matters, lots of work has explored paradigms and prototypes to overcome the shortcomings of distance and physical presence in decades. For example, in the 1990s, Kuzuoka et al have developed prototypes about video-mediated communication systems to stimulate spatiality and deliver emotional expression (e.g., Kuzuoka 1992; Kuzuoka et al. 1994). A group of researchers has focused on tangible interfaces to overcome the haptic limitation and physical background in graphical user interfaces and to enhance remote collaboration and communication (e.g., Ishii and Ullmer 1997; Brave et al. 1998). Research about gaze motion and gesture and shape capture and their implications on telecommunication and telepresence has been continuously discussed (e.g., Ishii et al. 1993; Fussell et al. 2003; Sirkin et al. 2011; Leithinger et al. 2014).

With the advancement of technological infrastructure, remote collaboration research has been involved in the mobile collaboration (Gauglitz et al. 2012), information visualization (Balakrishnan et al. 
2008), Augment Reality (AR) (Gauglitz et al. 2014a), Virtual Reality (VR) (Williamson et al. 2021), and Mix Reality (MR) (Piumsomboon et al. 2018; Teo et al. 2019). For example, Gauglitz et al. (2014b) has developed a live AR-based prototype allowing a remote user to mark annotations through a touchscreen to instruct and assist a field worker to solve problems. All these novel techniques are still at their early stage in lab or field research and not available at scale in commercialized remote collaboration platforms.

The current crisis increases society's reliance on technology with social and occupational changes (Garfin 2020). Many media reports show a surge of video conferencing app downloads for work collaboration and keeping in touch with family and friends in Europe and the US (Sydow 2020; Trueman 2020). The transformation from workspace to teleworking decreases the frequency of adhoc meetings due to the lack of spontaneity and further impedes knowledge sharing and coordination for knowledge workers (Waizenegger et al. 2020). The increased video conferencing meetings can induce fatigue and anxiety towards the overuse of technology (Wiederhold 2020). The technological exhaustion results from the technological shortcomings of video conferencing platforms such as lack of eye contact, and limited nonverbal cues (Riva et al. 2020). Wu and Chen (2020) found that the technology use also increases workload and decreases productivity and that the technical issues, such as less efficiency of online communication, are the core reasons. Though some work points out the limitations of this enforced teleworking context, there still lacks a focused and comprehensive study to explore teleworkers' perceptions of technology use for work collaboration during the pandemic. Thus, we asked:

- RQ1: What are teleworkers' perceptions of using technology to work and collaborate with others remotely during the pandemic?

As for social context, research proposes to use technology to improve the quality of our personal experience and to augment the existing strategies to generate psychological well-being; social technology such as $\mathrm{m}$-Health and smartphone apps, stand-alone and social virtual reality, video games, exergames, can potentially enhance our personal experience (e.g., emotion, engagement, and connectedness) that is challenged by the pandemic (Riva et al. 2020). For example, video games allow individuals to connect and socialize through play as a way to mitigate the psychological impact of the pandemic (Marston and Kowert 2020). Exergames are easy-to-use tools to cope with social isolation and maintain physical fitness during the quarantine (Viana and De Lira 2020). In general, video games not only provide ways to connect with others and maintain mental well-being, but also offer opportunities to substitute reality to host events and to find ways to gamify everyday activities (Kleinman et al. 2021). Research targeting aging populations' technology use found that older adults use various mobile technologies to stay connected with family (e.g., Facetime, Skype), maintain mobility, and link to resources helpful for their physical and psychological well-being (Banskota et al. 2020). While many media reports show the increased use of mobile technology and social media, and some research highlights the importance of different technologies that can potentially benefit the users, little user research explores teleworkers' attitudes and perceptions of the technology use in social context during the pandemic. Thus, we asked:

- RQ2: What are teleworkers' perceptions of using technology to socialize with others remotely during the pandemic?

\section{METHODS}

\subsection{Participant Recruitment}

We received approval from the Institutional Review Board (IRB) to carry out the project and interview protocol. We interviewed 46 participants who were adults from the United States who had to work from home due to the pandemic. The participants were recruited through a convenience sample and were family, friends, or loose acquaintances of our research assistants in the lab and 12 students taking a computing-related course. Most participants were young adults in their 20's (60.9\%) (M = 27.7 , range $=19$ to 55). There were 19 female participants $(41.3 \%)$ and 22 male participants (47.8\%). There were 23 Asian participants (50.0\%), 10 Caucasian participants $(21.7 \%), 5$ African American participants (10.9\%), and 3 Hispanic participants $(6.5 \%)$. Five participants, labeled as $\mathrm{N} / \mathrm{A}$, did not share their demographics (10.9\%). Demographic details and participant occupation details are summarized in theAppendix A.

\subsection{Interview Process}

Semi-structured interviews were conducted remotely via Skype, Discord, or FaceTime. They lasted for around 15 to 45 minutes. We started with fixed warm-up questions about their general remote living experience like "What does your current living situation look like?" and "Can you walk me through what you did yesterday?" Then, we asked questions more specific to our research questions (This part of the protocol is semi-structured with fixed questions 
related to work and socialization and potential followup questions based on participants' responses). We first asked questions about their remote work transition with questions like "Do you think the transition to work from home was easy or difficult?" and "Has this work environment affected your productivity?", following up with questions about their collaboration experience like "Has the collaboration method changed since the quarantine" and "How is the new method of collaboration impacting your productivity?" Next, we asked questions about their remote lifestyle transition with questions like "How often do you go out of your home?" and "What events have been canceled for you?", following up with questions about their socialization experiences like "How do you connect with family and friends virtually?" and "What are the pros and cons in streaming holiday parties and events?". Throughout the interview, we asked participants to elaborate on their thoughts and feelings. Finally, we asked general demographic questions about their age, race, gender, and occupation. We also opened the floor to any other related insights or experiences the participants may have had that were not covered during the interview.

\subsection{Interview Analysis}

Each interview recording was transcribed through speech transcription software. The research assistants then reviewed and familiarized themselves with each transcript. We used a thematic analysis to identify, classify, and analyze themes (Nowell et al. 2017) shared within participant's remote interaction experiences during the pandemic. To find these themes, we used an inductive coding process. Five researchers were involved in the analysis process (three for coding and organizing and two for calibration). First, three researchers organized participant's responses on a spreadsheet, based on their relevance to the research questions. Second, each researcher developed codes from the organized responses, which were presented and explained in detail during weekly meetings with the other two researchers. During these meetings, the team reviewed and discussed the codes and quotes together until all five researchers reached an agreement. This process was repeated until all codes were consistent and finalized for both research questions. Third, after the coding process was completed, these codes were imported into Miro, an online collaborative whiteboard tool. Each code became a sticky note on the whiteboard, with a tag that displayed which participant it belonged to. Three researchers worked together to group codes and presented themes found among the grouped codes with the other two researchers for calibration. Fourth, when the major themes were identified, we reviewed each theme's codes in-depth to find any sub-themes. All the themes and sub-themes were developed and agreed upon by the five researchers. Finally, quotes that best represented the themes were found and reported in the results section. This analysis process took several weeks to complete through many weekly meetings and discussions.

\section{RESULTS}

\subsection{Perceptions of Work Collaboration}

\subsubsection{Flatten Communication Hierarchy}

As an outcome of transitioning to online communication, participants developed more streamlined communication channels with others. Before the pandemic, P33 (M, 26), a software engineer, would ask questions or receive updates indirectly through his co-worker, who relayed the information from their boss. However, as a result of being remote, P33 now communicates with his boss, which he noted was a significant change in his collaboration. Similarly, P38 (M, 29), a university lecturer, found that teaching classes online through WebEx, provided more opportunities for his students to ask him private questions without interrupting the class, which was challenging to do in person. The straightforward nature of communication platforms allowed participants to communicate more directly with others, especially those higher on the chain of command.

\subsubsection{Extend Workload and Duration via Remote Collaboration}

Tasks that were more individually focused were suited to be done remotely; however, tasks that require collaboration were more difficult to do remotely in comparison, extending workload and completion time as a result. Being in the office naturally facilitates conversations among co-workers to solve any spontaneous problems or provide updates. However, because participants working from home needed to wait for responses or scheduled meetings to collaborate, it added more effort and time to complete tasks or notify others. P34 (F, 30), a corporate office manager for a car wash, found it challenging to stay connected with her team. It took extra work to set up multi-way calls and to call her team for every update: "If we were right next to each other, we could just say, 'Hey, this is what's going on today and that's it. But now it's a little different because things tend to pop up throughout the day where we need to call each other." Remote collaboration does not work as smoothly as an inperson collaboration due to the delay in reaction from others and additional effort to stay in touch.

\subsubsection{Blurs Time on and off-hours}

Working at the office allowed participants to create boundaries between work and personal life 
due to the physical distinction between the two locations. However, remote work solutions increased accessibility to work, blurring the lines between work and personal life for those who worked from home. As a result, participants were expected to work beyond their standard work hours, which interfered with their personal schedules: "I rarely received information from the team members at seven or eight in the evening, but now the information that needs to be responded to during this period has increased" (P30, M, 31). P17 (F, 55) was appreciated by her supervisor for putting more effort to be more accessible '24/7' to her students and parents during the pandemic.

\subsubsection{More Tracking and Planning Needed}

More planning and tracking were required to continue working from home. P46 (M, 23) is a network administrator, developer, and recruiter: "Arranging [meetings] for more than two people, including myself, to collaborate requires a little more planning compared to before, where I could just walk over to the desk of a colleague and see if they were busy and just start talking to them." Just to collaborate with others, extra planning and setup were needed, which slowed work efficiency. There were also more tools participants needed to keep track of in order to work and collaborate remotely.

\subsubsection{Technical Tools Delayed Productivity} Many adopted new technologies to work from home; however, managing multiple platforms became more difficult with the lack of experience using them. P29 (F, 26), a geometry teacher, felt that her job became more strenuous as a result of all the new tools she had to 'keep up with' to continue teaching online. Along with the limited experience using these technologies, technical difficulties also slowed work efficiency: "Sometimes it's faster when it is in-person because you are able to show the person additional information right in front of them. With online [tools], technical issues always seem to cause delays, such as getting the shared screen setup or someone talking while on mute" (P41, F, 26). Because participants had to find technological solutions at such short notice, the lack of expertise and training to use these tools made it difficult to work remotely.

\subsubsection{Lack of surveillance}

Participants reported a decrease in surveillance from their supervisors and coworkers, which created less pressure while doing their work: "There's no one right behind you that's looking at you constantly and making sure that you're doing work" (P16, M, 22). Some participants reacted to the lack of supervision and decreased pressure positively. P16 (M, 22) initially expected the transition to work remotely would bring more strict oversight. He was surprised to experience the opposite effect. As a result, P16 felt it allowed him to work at his own pace due to the decrease in pressure.

Others reacted to the lack of supervision negatively, feeling that their work was not adequately acknowledged. P20 (M, 24), a reliability engineer, found it difficult to stand out and be recognized for his work when his supervisors were not present. Similarly, P9 $(M, 31)$ expressed how he felt less appreciated and valued when his actions were not seen directly by his boss. While the lack of surveillance lightened the weight of expectations from supervisors, it also made it easier for many efforts to be overlooked and underappreciated. Some participants felt less motivated to stay on top of their work (P20, M, 24).

\subsubsection{Lack of Physical Presence}

\subsubsection{Lack of Proximity}

Participants missed being in the close presence of their co-workers. P1 (M, 21) is a receptionist at a dental office who was suddenly conscious of the lack of his co-workers' presence. He missed having them around, describing them as 'good' and 'fun'. Other participants similarly longed for face-to-face interactions with their coworkers and were unaccustomed to not having them around. While participants appreciate having the technology to continue meeting with their coworkers, online interactions could not substitute faceto-face interactions: "I thank God [that] we have this internet world that we could depend on, but you know, l'd like to see them face to face" (P4, M, 23). Working from home made distancing from coworkers inevitable.

\subsubsection{Lack of Spontaneous Collaboration}

Many participants preferred working in the office because being near other co-workers allowed for spontaneous collaboration. Through body language and casual conversation, participants could gauge when it was the right time to approach a coworker to facilitate an unscheduled meeting. These impromptu conversations in the office made it easier to get instant feedback and responses. It was difficult to replicate the same experience online because remote collaboration was very planned and structured, leaving little room for spontaneity. Remote participants needed to create meetings or wait for others to respond: "I guess asking for help and stuff is a little difficult because you have to set up an appointment or set a meeting with my boss and like whoever can help me and make sure that they're free. And if they're not free, I have to wait for them. Meanwhile in the office, if I saw they were free I could just ask them. It's a lot more difficult to collaborate" (P16, M, 22). Collaborating online slowed down people's ability to work out problems together and limited who could 
get involved. P29 (F, 26), a geometry teacher, got more work done collaborating with other teachers in-person compared to sending emails back and forth: "It's better and more efficient when teachers meet to collaborate on upcoming events because it takes less time and more people can participate." Overall, the lack of a method to spontaneously and seamlessly collaborate with others hindered participant's ability to work as efficiently.

\subsubsection{Lack of Idea Generation}

It became more difficult to develop new ideas and be creative due to the inability to freely exchange ideas and thought processes with others. This was further hindered by the limited time of scheduled meetings and wait time for responses from others. P32 (F, 33), is an attorney: "If it was purely online, I think it would be harder to figure out creative solutions to problems because you don't have the back and forth that a conversation would allow you to, uh, not have the bounds." Because meetings are more planned, they are limited in allowing extra time to expand on ideas or conversations. P7 (F, 22), a process engineer, found that meetings became more straight to the point, making it difficult to develop ideas further or consider alternatives when the team was set on a particular solution.

\subsubsection{Lack of Emotional Expression and Shared Understanding}

Body language and emotional cues were difficult to communicate online, which hindered the participant's ability to monitor their coworker's reactions as feedback. P30 (M, 31) used his coworkers' expressions to guide decisions and check if his team was being productive: 'It's hard to feel everyone's emotions through remote video... I need to always pay attention to everyone's reactions, which will make it easier for me to make decisions." Others, like P41 $(F, 36)$, lost the ability to use reactions to measure whether the team needed more elaboration on material: "Since we do not do video conference for meetings, you do lose the ability to see people reactions. By reading certain people's faces in meetings I am able to potentially expand on certain subject matters within the meetings." Overall, online collaboration tools could not translate nonverbal communication smoothly, like body movements or facial expressions. It puts participants at a disadvantage because it was a viable source of information they could use to help them collaborate.

\subsubsection{Lack of Clarity of Expression via Video}

Video calls made it difficult to clearly express and convey information to others during meetings. For example, current remote work solutions were limited in functionality to support those, like P46 (M, 23), who visually express themselves or are more hands-on: "I'm a really visual person and hands-on, so already going completely online kind of puts me at a disadvantage really. So when I'm explaining something to someone, it gets a little harder to communicate exactly what you need when you're doing virtually versus just writing or drawing it out." Before the pandemic, P21's (F, 22) mentor at a technology co-op spoke directly to her and personally explained projects and tasks. This allowed P21 to understand the material at her own pace and guide the conversation to her needs. However, when meetings were online, she found it difficult to follow the conversation when the calls became more generalized and indirect.

4.1.5. Meetings are Structured instead of Free-form As a result of participant's limited time with other coworkers, meetings had become more structured to ensure work needs were met. P17 $(F, 55)$, a high school science teacher, described how teacher meetings with administration transformed from being free-formed group work and discussion to prepared agendas and presentations. Having more free-form meetings provided a fresh experience for the same work: "That's always changing. Like there are people to talk to. It's more spontaneous. Like I can go up to another co-worker and be like, hey, what's up? How are you doing? Sometimes they will have impromptu meetings so while the tasks are the same, the spontaneous gave something new" (P16, M, 22). These face-to-face interactions were opportunities to socialize and build work relationships. It is challenging to facilitate similar casual interactions when an online interaction is very planned.

\subsection{Perceptions of Socialization}

\subsubsection{Appreciating Seeing Faces and Talking Virtually But Still Missing Aspects of Physical Presence}

In relation to the pandemic, participants appreciated having online socialization methods available that helped them stay connected with others without needing to leave their homes. P26 (F, 49) did virtual workout calls every day with her friends and found it to be a positive and motivating experience. She enjoyed being in her friends' company, despite being apart. Participants P43 (N/A, N/A) and P46 (M, 23) were more optimistic considering the lack of an alternative, with P43 in particular sharing that, 'having something is always better than nothing.' However, while many had positive remote social experiences, participants still felt that some aspects of in-person interaction cannot be fully replaced by technology. Online socialization temporarily satisfied people's need to socialize with others, but was not a long-term solution.

While participants could see and communicate with others online, some aspects of in-person interaction 
were more challenging to convey through the screen, like physical presence and immersion. Many tried to make the most of the experience, however, it was difficult to enjoy virtual social events to the same extent as in-person events. There was a lack of physical presence when interacting with others digitally. $P 9(M, 31)$ compared the presence of others with talking to plastic: "And cons is, you feel physical plastic, you're not there with people, you can't celebrate with them directly... It's not the same." Body language was also difficult to communicate online through a webcam, making it harder to measure whether someone was engaged in the conversation.

Socializing remotely does not feel completely immersive. Participant P1 (M, 21) enjoyed social events at the cost of not being fully immersed in the experience and environment. There was also a lack of expected preparation that comes with online socialization that contributes to its immersion. P2 (F, 22) found that not dressing up took the fun away from attending events. It was harder for participants to 'live in the moment' without having a completely immersive experience.

\subsubsection{Accepting Limits of Main Social Media and Exploring Novel Ways}

Despite the limitations of social technology, participants used it to their advantage to improvise new ways to spend time with family and friends.

\subsubsection{Novel Apps to Socialize and Entertain}

Participants used this time as an opportunity to explore and experiment with the diverse portfolio of so- cialization technology available to find unique ways to interact with friends and family. Simply having conversations on online communication apps was no longer the only option people had to socialize. Novel gaming apps, like JackBox, Houseparty, and GamePigeon, became more popular amongst par- ticipants to use during social hangouts with others because the gaming element added an extra layer of engagement to the experience. Many enjoyed having a more exciting method to interact and connect with others while social distancing.

\subsubsection{Forgiving about Cancellations}

Participants were more forgiving about event cancellations as a result of a shared duty to follow social distancing regulations and protect their own and others' safety. Many recognized that there will always be more opportunities to celebrate and attend events after the pandemic. P20 (M, 24), in particular, had a more positive outlook despite losing the experience of an in-person graduation ceremony for his daughter: "I was even saving my sick days to be able to take off to go see her but it's okay, life is more important. We can celebrate these things later on." Participants also adopted a cautionary mentality when approaching these cancellations, preferring to be 'safe than sorry' ( $P 1, M, 21)$. Though participants were upset and disappointed, there were 'good reasons to cancel' (P7, F, 22) and it 'made sense'.

While there were virtual substitutes for these canceled events, participants were less inclined to attend because certain elements of the in-person experience could not be replicated. P15 $(F, 24)$ felt less motivated to attend an online concert because it was not as full and immersive. However, participants were still accepting of these virtual alternatives because they were better than having nothing ( $P 10$, $M, 46)$.

\subsubsection{Exploring New Ways to Socialize}

Participants utilized the available communication and socialization platforms to explore new ways they could host social events online. They celebrated virtual events like birthdays, happy hours with coworkers, and baby showers, among others. P32 (F, 33) and P27 (M, 35) both attended virtual funerals. P32 attended a Shivah that was streamed on Zoom to pay respects to her friend's grandmother. P27 felt that the virtual format for the Jewish funeral actually helped people reflect on the positive: "It was really focused on saying good stories of her. And just then a bunch of people was laughing because they were funny and happy stories about her. So I think it was better than a normal funeral... People were happy because they were remembering good times."

Some participants celebrated religious holidays virtually as well. P6 (M, 23) celebrated Ramadan through virtual streams held by mosques. P32 (F, 33) celebrated Seder with her family for Passover on Zoom. P13's (F, 23) family used FaceTime to help her celebrate the Tamil New Year tradition, despite living away from home: "What they did was at 6:30 AM, they FaceTimed me. I had to somehow walk out of my room and into the sofa out here, eyes closed, literally, eyes closed, walked out, and somehow answer the FaceTime call, and I had to look at this and so that they flip the camera around so that, that's still the first thing that I saw would be myself. They set up the mirror and everything back in Edison and they did that and I still kind of appreciate it because they're still trying to do what normal traditions are."

Some participants needed to get creative and improvise their technology use and their physical space for certain events. For her daughter's virtual ballet class, P42 (F, 33) used Zoom and accommodated her living space to make a makeshift ballet studio: "For my daughter's ballet lesson, it was done through Zoom. We hooked it up to the TV in the living room so that she could have a big screen for it. I didn't want her to feel like she couldn't move too 
much and not be able to see the screen anymore. It worked out perfectly... Luckily we were able to figure out the TV situation for our daughter. We had never used it that way before. So it all worked out."

\subsubsection{Relieves Some Concerns About Loved Ones Well-being}

Participants were remotely communicating with family members and friends more often to check their well-being. While P15 (F, 24) lived alone before the pandemic, she had concerned family members contacting her often to see how she was doing. Others used these checkups to catch up and stay updated in each other's lives. These periodic wellbeing checkups helped relieve concerns about loved ones and were often the main motive for contacting them. However, there can be a technological divide between the younger and older generations.

\subsubsection{Lower Barrier to Participate in Remote Socialization}

Due to the quarantine and stay-at-home recommendations, there was a lower barrier for participants to join online social events with family and friends. More people could attend events because the online format saved commuting time, created fewer excuses to not attend, and was easier to arrange. Participants were also able to have more frequent and longer social events. P44 (F, 21) had longer back-to-back events with friends because they were all at home with nothing else to do. While she had organized online events before the pandemic, it would take longer to arrange due to delayed communication. Some participants felt that the stay-at-home situation allowed for more control over scheduling and for better availability. P44 (F, 21) was more motivated to reach out to friends because the quarantine provided a 'break' to do so. P45 (F, 21) similarly had more free time to check up on friends often, which improved her relationships with them. The increased accessibility of online communication and socialization tools, combined with people's increased availability brought by the pandemic, created more opportunities to socialize in a time where in-person activity was limited.

\subsubsection{Facilitate Communication Among Those Who Don't Talk Regularly}

Participants reported reconnecting with those whom they don't talk with regularly. P44 (F, 21) had tried to stay in touch with her longtime high school friends in the past but found it difficult to arrange a time within their busy schedules. The quarantine provided an opportunity for P44 to have a reunion with her friends as a result of the extra free time. P27 (M, 35) connected and networked with new people through a Zoom birthday party. The quarantine became a chance for many to rekindle old relationships or start new ones. Participants found themselves communicating more with others in general. By continually checking up on her family's well-being, P42 (F, 33) contacted her family much more often than before. P33 (M, 26) talked more with his parents and his extended family, like his aunts and uncles, who all live abroad. This does not only pertain to close family and friends; $P 45(F, 21)$ found herself generally talking more with acquaintances through classes. Whether they have communicated often before the pandemic or not, participants found themselves initiating remote contact more often with family members, friends, or acquaintances in general.

\section{DISCUSSION}

This study could provide potential answers to recent research proposing to understand the adaption and impact of technology use during the pandemic by utilizing comparative analysis and to evaluate how teleworkers interact with technology and adapt to overcome contextual shortcomings (Dey et al. 2020).

By comparing the user's perceptions of technology use for work collaboration with their perceptions of technology use for personal socialization, we found that users generally had more positive experiences and attitudes for using social technologies. Users were understanding of the technological limitations and to some extent appreciated the availability of social technology, trying to find new online social platforms or re-purpose existing ones. However, users showed more negative attitudes and had less positive experiences with using collaborative technology. Users expressed strong concerns about the lack of physical presence and workload caused the technological limitations, though online communication flattens the communication hierarchy. Additionally, the overlap of concern between work and social technology use was the lack of physical presence, but it was a bigger concern in work collaboration than socialization. Possibly, task-oriented and relationshiporiented communication is the key difference. How the purpose of communication affects user's perception of technology use might be an interesting topic to explore for future research.

The pandemic restrains user's mobility and affects their behaviors that normally have to do face-to-face. Users mixed the work and social technology use to meet their needs and overcome the shortcomings of not being able to gather together. For example, users reported using FaceTime to celebrate normal traditions with family remotely and attending funerals on Zoom, scenarios that have rarely happened online and neglected by designers in the prepandemic. The novel experience might reduce their 
negative feelings and make them still consider these technologies helpful in general.

COVID-19 not only causes physical health concerns but also results in a number of mental health disorders such as stress, depression, and anxiety (Salari et al. 2020; Brooks et al. 2020). Psychological interventions related to providing social support are essential to preserving mental health, such as staying with family to relieve people's anxiety caused by the crisis (Cao et al. 2020; Son et al. 2020). Mental health professionals also suggest individuals use alternative communication methods such as social networks and digital communication platforms to cope with social isolation (Banerjee 2020), and propose to scale up mental health services and support through mobile technology interventions such as apps and text messaging (Figueroa and Aguilera 2020). We contextualize these suggestions and show that users appreciate the available social technologies to connect with family and friends, relieve their concerns, and interestingly, promote conversation among those who do not talk regularly. Is it a way to cope with anxiety, fear of death, or social isolation? What's the psychological logic behind this perception? It also raises questions about whether the crisis can impose communication and the potential consequences.

Teleworking in the pre-pandemic was described as "technologically feasible, flexible and autonomous, desirable and perhaps even inevitable, family- and community-friendly" (Bryant 2000). It seems that forced teleworking diminishes the benefits that are usually perceived in a regular/voluntary context. The overall negative attitude towards technology use in work collaboration can be in line with Olson and Olson's work (Olson and Olson 2000).

The pandemic makes people unable to be ready for collaboration. The new normal working styles need time to learn how to collaborate remotely, especially those who have not been working from home. The collaborative technology is still not ready to cope with the need to fully work from home as many users reported a lack of physical presence for the existing technologies. The homogeneous collaboration tools on the market still suffer many limitations that have been discussed and existed for decades (Ishii 1990; Kuzuoka et al. 1994; Ishii and Ullmer 1997). Though technology use increases, the dependency/ coupling of work is still strong. Typically, the strong dependency of work requires frequent and complex communication with short feedback loops and is usually achieved through collocated interaction (Olson and Olson 2000). The physical distancing and forced teleworking further amplify these challenges.
Common ground can be easily established in a collocated environment because people can share not only cultural and local context but also the more micro context of who is doing what at the moment and what remains to be done, and teleworking makes the establishment challenging because a variety of cues are lost (Olson and Olson 2000). We do not specifically address whether the users shared a common ground during the collaboration process. The issues (e.g., lack of clarity of expression, emotional expression, and understanding) might infer the difficulty to build common ground for teleworking collaboration, requiring further investigation.

Users showed mixed attitudes towards lack of surveillance, which, on one hand, reduced pressure from their supervisors; while, on the other hand, generated less appreciation from their supervisors. When the camera is not turned on, teleworkers' effort and progress were invisible to their peers and supervisors. These mixed attitudes support prior work stating that there is only a thin line of difference in perceiving the same technology for surveillance or for assessment (Das Swain et al. 2020). How to balance the support and surveillance of teleworkers should be evaluated further.

\subsection{Design Implications}

\subsubsection{More Immersive and Embedded Environment of Collaborative Work}

Many cues that are usually available in the workplace were missing from the forced teleworking. Though teleworkers can communicate and see faces virtually, the nuanced cues in physical environments are missed (e.g., social banter and buzz that constitutes an office environment (Mann and Holdsworth 2003)). A recent report also shows that being face-to-face is important not only for "getting the job done" but also for enjoying work more generally (Rubin et al. 2020).

The critical key to timely completing tasks is the fluidity of participation that allows one or two team members to work alone while others have a spontaneous meeting, team members moving from one subgroup to another or the team having a meeting as a whole (Olson and Olson 2000). The live synchronization of VR technology with more interactive tools/elements such as spatial audio, haptic feedback, 360 video streaming, and motion capture can potentially overcome the limitations of current communication systems in work collaboration (Neurons 2017; Huang et al. 2020). For example, is it possible to design a live VR meeting system with spatiality to simulate a virtual distance and facilitate the fluidity of online participation? The users can choose who to virtually 'sit' close with 
and can have proximal interaction to stimulate the distance in the workplace. While someone is talking to close colleagues, it won't disrupt other colleague's performance, mimicking the social buzz in the physical environment. VR can also provide more cues to facilitate the common ground construction, reducing misinterpretations and the following work to repair. The Limitation of the head-mounted-display equipment in VR might also suggest that $A R$ or MR technologies can have much more opportunities for innovation. We also want to clarify that these technologies were discussed early in research but have not been commercialized in a long time. The pandemic shows the necessity to consider feasible ways to make them available at scale.

\subsubsection{Tools to Facilitate Peer Support and \\ Self-evaluation and to Track Work Progress and Performance}

Teleworking limits interactions with co-workers, and also makes it difficult to self-evaluate their performance and be aware of other co-workers' expectations during team collaboration (Das Swain et al. 2020). Nicholas Bloom (2020) suggests that an effective "performance review system" to evaluate teleworkers' output is essential to make teleworking succeed. Wu and Chen (2020) propose that $\mathrm{HCl}$ work should support on-site work and lab-based tasks to improve the feasibility of teleworking. In line with the thread, designers can design a collaborative tool to monitor user's work progress (not only the output) by checking in milestones and facilitate peer support so that co-workers can coordinate their tasks and normalize their routines to others', the supervisor can evaluate teleworkers' performances by not only the output but also the progress.

\subsubsection{More Situated Elements for Socializing}

Because users were more creative with social technology use and social activities were more fluid, there are opportunities to design tools that better support different social contexts. We exemplify that the users applied various technologies to socialize with others and repurposed them to fulfill their social needs, situations that were not envisaged by the designers pre-pandemic (Dey et al. 2020). Future designs should consider how to stimulate and facilitate pre-pandemic physical group activities online. For example, is there a better way to support those attending funerals with family and friends with more engaging and emotionally connected tools?

\subsection{Limitations and Future Work}

This is a preliminary study about teleworkers' perceptions of technology use for work collaboration and socialization during the pandemic. It suffers several limitations. First, we used a convenience sample, so our findings may not be representative of all teleworkers. Future research should apply other sampling methods to increase the representation of the sampling. Additionally, the demographics of our samples show diverse professions. The pandemic affects different occupations differently. Future work can focus on a specific group and explore their perceptions in-depth. Second, our participants were individuals from the US and do not represent those from other countries, who may have faced different impacts and policies caused by the pandemic. The technological tools used for work collaboration and socialization may differ in other countries as well. Future work could involve a crosscountry study to explore teleworkers' practices and perceptions across different cultural backgrounds and technological availability. Third, a majority of our participants were young adults, those who are older or not as technologically savvy were not wellrepresented. Future work could extend this study to explore older adults' perceptions toward technology use during the pandemic. Fourth, teleworking is also highly relevant to the family context, which was not focused on in this exploratory study. Future work can also consider exploring how the environment and family conditions affect their perceptions of socializing and working. Lastly, the data collection was completed in the early stage of the pandemic. It is an unusual situation for the participants to use technology for teleworking, where they might have experienced difficulty in using technology. Nowadays, they may have adapted to the technology use and changed their behaviors and perceptions towards the technology. Future work can also explore the dynamics of the perception of technology use during the pandemic.

\section{CONCLUSION}

This study aims to understand teleworkers' perceptions of technology use in work collaboration and socialization. We found that the challenges in work collaboration still exist and are amplified by the pandemic, though prior research has raised these questions and provided potential solutions. Users are creative to explore new technologies or re-purpose available technologies to meet social needs. Designers for telecommunication platforms should consider the implications of prototypes in prior work and find feasible ways to commercialize them, and designers should take the scenarios that are typically missed in social-technical design in the pre-pandemic into consideration.

\section{ACKNOWLEDGMENTS}

Thanks to Daniel for the assistance of data analysis and the research assistants from SocialXLab for data collection. 


\section{REFERENCES}

Balakrishnan, A. D., S. R. Fussell, and S. Kiesler (2008). Do visualizations improve synchronous remote collaboration? In Proceeding of the twentysixth annual $\mathrm{CHI}$ conference on Human factors in computing systems - CHI '08, New York, New York, USA, pp. 1227. ACM Press.

Banerjee, D. (2020, 4). The COVID-19 outbreak: Crucial role the psychiatrists can play.

Banskota, S., M. Healy, and E. M. Goldberg (2020). 15 smartphone apps for older adults to use while in isolation during the Covid-19 pandemic.

Brave, S., W. Khii, and M. Hdre (1998). Tangiblelnterfaces for Remote Collaborationand Communication. In Proceedings of the ACM Conference on Computer Supported Cooperative Work, pp. 10.

Brooks, S. K., R. K. Webster, L. E. Smith, L. Woodland, S. Wessely, N. Greenberg, and G. J. Rubin (2020). The psychological impact of quarantine and how to reduce it: rapid review of the evidence.

Bryant, S. $(2000,3)$. At home on the electronic frontier: work, gender and the information highway. New Technology, Work and Employment 15(1), 19-33.

Cao, W., Z. Fang, G. Hou, M. Han, X. Xu, J. Dong, and J. Zheng (2020). The psychological impact of the COVID-19 epidemic on college students in China. Psychiatry Research287.

Collins, M. (2005, 7). The (not so simple) case for teleworking: a study at Lloyd's of London. New Technology, Work and Employment 20(2), 115132.

Cox, A. L., S. Dray, J. Bird, A. Peters, N. Mauthner, and E. Collins (2014). Socio-technical practices and work-home boundaries. In Mobile $\mathrm{HCl} 2014$

- Proceedings of the 16th ACM International Conference on Human-Computer Interaction with Mobile Devices and Services, pp. 581-584.

Das Swain, V., K. Saha, G. D. Abowd, and M. De Choudhury (2020). Social media and ubiquitous technologies for remote worker wellbeing and productivity in a post-pandemic world. In Proceedings - 2020 IEEE 2nd International Conference on Cognitive Machine Intelligence, CogMI 2020, pp. 121-130.

Dey, B. L., W. Al-Karaghouli, and S. S. Muhammad (2020). Adoption, Adaptation, Use and Impact of Information Systems during Pandemic Time and
Beyond: Research and Managerial Implications. Information Systems Management 37(4), 298302.

Figueroa, C. A. and A. Aguilera $(2020,6)$. The Need for a Mental Health Technology Revolution in the COVID-19 Pandemic. Frontiers in Psychiatry 11.

Fonner, K. L. and L. C. Stache (2012). All in a day's work, at home: Teleworkers' management of micro role transitions and the work-home boundary. New Technology, Work and Employment 27 (3), 242257.

Fussell, S. R., L. D. Setlock, and R. E. Kraut (2003). Effects of head-mounted and sceneoriented video systems on remote collaboration on physical tasks. In Proceedings of the conference on Human factors in computing systems - $\mathrm{CHI}$ '03, New York, New York, USA, pp. 513. ACM Press.

Fussell, S. R., L. D. Setlock, J. Yang, J. Ou, E. Mauer, and A. D. Kramer (2004). Gestures over video streams to support remote collaboration on physical tasks. Human-Computer Interaction 19(3), 273-309.

Gajendran, R. S. and D. A. Harrison (2007). The Good, the Bad, and the Unknown About Telecommuting: Meta-Analysis of Psychological Mediators and Individual Consequences. Journal of Applied Psychology 92(6), 1524-1541.

Garfin, D. R. (2020, 10). Technology as a coping tool during the coronavirus disease 2019 (COVID-19) pandemic: Implications and recommendations. Stress and Health 36(4), 555-559.

Gauglitz, S., C. Lee, M. Turk, and T. Höllerer (2012). Integrating the physical environment into mobile remote collaboration. In Proceedings of the 14th international conference on Human-computer interaction with mobile devices and services Mobile HCl '12, New York, New York, USA, pp. 241. ACM Press.

Gauglitz, S., B. Nuernberger, M. Turk, and T. Höllerer (2014a). In touch with the remote world: Remote collaboration with augmented reality drawings and virtual navigation. In Proceedings of the ACM Symposium on Virtual Reality Software and Technology, VRST, pp. 197-205.

Gauglitz, S., B. Nuernberger, M. Turk, and T. Höllerer (2014b). World-stabilized annotations and virtual scene navigation for remote collaboration. UIST 2014 - Proceedings of the 27th Annual ACM Symposium on User Interface Software and Technology , 449-460. 
Golinelli, D., E. Boetto, G. Carullo, A. G. Nuzzolese, M. P. Landini, and M. P. Fantini (2020, 11). Adoption of Digital Technologies in Health Care During the COVID-19 Pandemic: Systematic Review of Early Scientific Literature. Journal of Medical Internet Research 22(11), 23.

Huang, H. Y., C. W. Ning, P. Y. Wang, J. H. Cheng, and L. P. Cheng (2020). Haptic-go-round: A Surrounding Platform for Encounter-type Haptics in Virtual Reality Experiences. In Conference on Human Factors in Computing Systems Proceedings, pp. 10.

Ishii, H. (1990). Team workstation: Towards a seamless shared workspace. In Proceedings of the 1990 ACM Conference on ComputerSupported Cooperative Work, CSCW 1990, New York, New York, USA, pp. 13-26. ACM Press.

Ishii, H., M. Kobayashi, and J. Grudin (1993). Integration of Interpersonal Space and Shared Workspace: ClearBoard Design and Experiments. ACM Transactions on Information Systems (TOIS) 11(4), 349-375.

Ishii, H. and B. Ullmer (1997). Tangible bits: Towards seamless interfaces between people, bits and atoms. In Conference on Human Factors in Computing Systems - Proceedings, pp. 234-241.

Kissler, S. M., C. Tedijanto, E. Goldstein, Y. H. Grad, and M. Lipsitch $(2020,5)$. Projecting the transmission dynamics of SARS-CoV-2 through the postpandemic period. Science 368(6493), 860-868.

Kleinman, E., S. Chojnacki, and M. S. El-Nasr (2021). The Gang's All Here: How People Used Games to cope with COVID19 Quarantine. In Proceedings of $2021 \mathrm{CHI}$ Conference on Human Factors in Computing Systems - CHI ' 2021.

Kuzuoka, H. (1992). Spatial workspace collaboration: a sharedview video support system for remote collaboration capability. In Conference on Human Factors in Computing Systems - Proceedings, pp. 533-540.

Kuzuoka, H., T. Kosuge, and M. Tanaka (1994). GestureCam: A video communication system for sympathetic remote collaboration. In Proceedings of the 1994 ACM Conference on Computer Supported Cooperative Work, CSCW 1994, New York, New York, USA, pp. 35-43. ACM Press.

Leithinger, D., S. Follmer, A. Olwal, and H. Ishii $(2014,10)$. Physical Telepresence: Shape capture and display for embodied, computer-mediated remote collaboration. In UIST 2014 - Proceedings of the 27th Annual ACM Symposium on User
Interface Software and Technology, pp. 461-470. Association for Computing Machinery, Inc.

Liu, J., W.-H. Cheng, K. Nahrstedt, R. Jain, E. Ricci, and $H$. Byun (2020). Coping with Pandemics: Opportunities and Challenges for Al Multimedia in the "New Normal". In ACM International Conference on Multimedia (MM '20), pp. 47944795. ACM.

Mann, S. and L. Holdsworth $(2003,11)$. The psychological impact of teleworking: stress, emotions and health. New Technology, Work and Employment 18(3), 196-211.

Marston, H. R. and R. Kowert $(2020,6)$. What role can videogames play in the COVID-19 pandemic? Emerald Open Research 2, 34.

Maruyama, T., P. G. Hopkinson, and P. W. James (2009). A multivariate analysis of work-life balance outcomes from a large-scale telework programme. New Technology, Work and Employment 24(1), 76-88.

Mattern, J., S. Lansmann, and J. A. Hüllmann (2021). It's not that bad! Perceived Stress of Knowledge Workers During Enforced Working From Home due to COVID-19. In Proceedings of the 16th International Conference on Wirtschaftsinformatik (WI), Number March.

Nah, F. F. H. and K. Siau (2020). Covid-19 pandemic - role of technology in transforming business to the new normal. Lecture Notes in Computer Science (including subseries Lecture Notes in Artificial Intelligence and Lecture Notes in Bioinformatics) 12427 LNCS, 585-600.

Neurons (2017). How virtual reality facilitates social connection.

Nicholas Bloom (2020). How working from home works out. Stanford, Institute for Economic Policy Research (SIEPR) (June), 8.

Nowell, L. S., J. M. Norris, D. E. White, and N. J. Moules (2017). Thematic Analysis: Striving to Meet the Trustworthiness Criteria. International Journal of Qualitative Methods 16(1).

Olson, G. M. and J. S. Olson (2000). Distance Matters. Human -Computer Interaction 15(2-3), 139-178.

Piumsomboon, T., G. A. Lee, J. D. Hart, B. Ens, R. W. Lindeman, B. H. Thomas, and M. Billinghurst (2018). Mini-me: An adaptive avatar for Mixed Reality remote collaboration. Conference on Human Factors in Computing Systems Proceedings 2018-April. 
Riva, G., F. Mantovani, and B. K. Wiederhold $(2020,9)$. Positive technology and COVID-

19. Cyberpsychology, Behavior, and Social Networking 23(9), 581-587.

Rubin, O., A. Nikolaeva, S. Nello-Deakin, and M. t. Brommelstroet (2020). What can we learn from the COVID-19 pandemic about how people experience working from home and commuting? Technical report, University of Amsterdam.

Saladino, V., D. Algeri, and V. Auriemma (2020). The Psychological and Social Impact of Covid19: New Perspectives of Well-Being. Frontiers in Psychology 11,2550.

Salari, N., A. Hosseinian-Far, R. Jalali, A. VaisiRaygani, S. Rasoulpoor, M. Mohammadi, S. Rasoulpoor, and B. Khaledi-Paveh (2020). Prevalence of stress, anxiety, depression among the general population during the COVID-19 pandemic: A systematic review and meta-analysis. Globalization and Health 16(50), 11.

Sirkin, D., G. Venolia, J. Tang, G. Robertson, T. Kim, K. Inkpen, M. Sedlins, B. Lee, and M. Sinclair (2011). Motion and attention in a kinetic videoconferencing proxy. In Lecture Notes in Computer Science, pp. 162-180. Springer, Berlin, Heidelberg.

Son, C., S. Hegde, A. Smith, X. Wang, and

F. Sasangohar (2020). Effects of COVID-19 on college students' mental health in the United States: Interview survey study. Journal of Medical Internet Research 22(9).

Stawarz, K., A. L. Cox, J. Bird, and R. Benedyk (2013). "I'd Sit at Home and Do Work Emails": How Tablets Affect the Work-Life Balance of Office Workers. In Proceedings of the ACM Conference on Human Factors in Computing Systems, pp. 1383-1388.

Sydow, L. (2020). Video Conferencing Apps Surge from Coronavirus Impact.

Teo, T., L. Lawrence, G. A. Lee, M. Billinghurst, and M. Adcock (2019). Mixed reality remote collaboration combining 360 video and 3D reconstruction. In Conference on Human Factors in Computing Systems - Proceedings, pp. 14. ACM.

Trueman, C. (2020). Pandemic leads to surge in video conferencing app downloads.

Viana, R. B. and C. A. B. De Lira (2020). Exergames as Coping Strategies for Anxiety Disorders during the COVID-19 Quarantine Period. Games for Health Journal 9(3), 147-149.

Waizenegger, L., B. McKenna, W. Cai, and T. Bendz $(2020,7)$. An affordance perspective of team collaboration and enforced working from home during COVID-19. European Journal of Information Systems 29(4), 429-442.

Wiederhold, B. K. (2020, 7). Connecting through Technology during the Coronavirus Disease 2019 Pandemic: Avoiding "zoom Fatigue". Cyberpsychology, Behavior, and Social Networking 23(7), 437-438.

Williamson, J. R., J. Li, V. Vinayagamoorthy, D. A. Shamma, and P. Cesar (2021). Proxemics and Social Interactions in an Instrumented Virtual Reality Workshop. In Proceedings of $2021 \mathrm{CHI}$ Conference on Human Factors in Computing Systems - CHI' 2021.

Wold, S. (2021, 9). COVID-19 is changing how, why and how much we're using social media. Technical report.

Wu, H. and Y. Chen $(2020,7)$. The impact of work from home (wfh) on workload and productivity in terms of different tasks and occupations. In HCl International 2020 - Late Breaking Papers: Interaction, Knowledge and Social Media. HCll 2020. Lecture Notes in Computer Science, Volume 12427, pp. 693-706. Springer, Cham.

\section{A. APPENDIX: DEMOGRAPHIC AND INDUSTRY OF PARTICIPANTS}




\begin{tabular}{|c|c|c|c|c|}
\hline ID & $\begin{array}{l}\text { Occupation \& Industry } \\
\text { Receptionist at a Dental Office }\end{array}$ & $\begin{array}{l}\text { Age } \\
21\end{array}$ & $\begin{array}{l}\text { Race } \\
\text { White }\end{array}$ & $\begin{array}{r}\text { Gender } \\
\text { Male }\end{array}$ \\
\hline P1 & $\begin{array}{l}\text { Receptionist at a Dental Office } \\
\text { Customer Services Renresentative }\end{array}$ & 21 & White & Male \\
\hline P2 & Customer Services Representative & 22 & White & Female \\
\hline P3 & Scheduling Manager & 19 & Asian & Male \\
\hline P4 & E-trader & 23 & White & Male \\
\hline P5 & Medical Advisor & $N / A$ & N/A & $\mathrm{N} / \mathrm{A}$ \\
\hline P6 & Assistant Project Manager & 23 & White & Male \\
\hline P7 & Process Engineer & 22 & Asian & Female \\
\hline P8 & Emergency Medical Technician & 19 & Asian & Male \\
\hline P9 & Desk Consultant and Fitness Employee & 31 & Hispanic & Male \\
\hline P10 & Library Staff & 46 & African American & Male \\
\hline P11 & Graphic Design Intern & 21 & Asian & Female \\
\hline P12 & Cybersecurity Technology At a Bank & 51 & Asian & Male \\
\hline $\mathrm{P} 13$ & Pediatric Newborn Research Assistant & 23 & Asian & Female \\
\hline P14 & Programmer \&amp; Business Owner & 29 & Asian & Male \\
\hline P15 & Production Engineering Intern & 24 & Asian & Female \\
\hline P16 & Data Analyst & 22 & Asian & Male \\
\hline P17 & High School Science Teacher & 55 & Asian & Female \\
\hline P18 & Software Developer & 25 & White & Male \\
\hline P19 & Coding Teacher & 21 & Asian & Male \\
\hline P20 & Reliability Engineer & 24 & Asian & Male \\
\hline P21 & Technology Co-op & 22 & Asian & Female \\
\hline P22 & Student & 23 & Asian & Female \\
\hline P23 & Unemployed & 29 & White & Male \\
\hline P24 & Senior Research Analyst in Healthcare & 26 & African American & Male \\
\hline P25 & RF Engineer for Corporate & 27 & Asian & Male \\
\hline P26 & Insurance Agent & 49 & Hispanic & Female \\
\hline P27 & Small Startup & 35 & White & Male \\
\hline P28 & Talent Coordinator & 23 & African American & Female \\
\hline P29 & Geometry Teacher & 26 & African American & Female \\
\hline P30 & Software Engineer & 31 & Asian & Male \\
\hline P31 & Software Engineer & 26 & Asian & Female \\
\hline P32 & Attorney & 33 & White & Female \\
\hline P33 & Software Engineer & 26 & Asian & Male \\
\hline P34 & Corporate Office Manager for Car Wash & 30 & White & Female \\
\hline P35 & Student & $N / A$ & N/A & $\mathrm{N} / \mathrm{A}$ \\
\hline P36 & Product Analyst & $N / A$ & $\mathrm{~N} / \mathrm{A}$ & $\mathrm{N} / \mathrm{A}$ \\
\hline P37 & Student & 22 & Asian & Female \\
\hline P38 & University Lecturer & 29 & White & Male \\
\hline P39 & Ph.D. student & 25 & Asian & Male \\
\hline P40 & Product Owner in Customer Technology Team & $N / A$ & N/A & $N / A$ \\
\hline P41 & Manager in Risk Adjustment for Healthcare Company & 36 & African American & Female \\
\hline P42 & Senior Tax Reporting and Company Analyst & 33 & Hispanic & Female \\
\hline P43 & Masters Student & $N / A$ & N/A & $\mathrm{N} / \mathrm{A}$ \\
\hline P44 & Student \& Dance Teacher & 21 & Asian & Female \\
\hline P45 & Student \& Substitute & 21 & Asian & Female \\
\hline P46 & Network Administrator, Developer, Recruiter & 23 & Asian & Male \\
\hline
\end{tabular}

